\title{
Analysis of Immunogenicity data in the Product Information of Biological drugs: A need to report immunogenicity data systematically
}

Rodrigo Borrega ${ }^{1}, \ldots . .$. Joao Goncalves ${ }^{1^{*}}$

${ }^{1}$ iMed- Research Institute for Medicines, Faculty of Pharmacy at University of Lisbon, Lisbon, Portugal

\section{* Correspondence:}

Joao Goncalves

Faculdade de Farmácia Universidade Lisboa

iMed.ULisboa - Research Institute for Medicines

Av. Prof. Gama Pinto, 1649-019 Lisboa

Portugal

Phone: $+351217946486 /+351217946489$

e-mail: joao.goncalves@ff.ul.pt

ORCID: 0000-0002-1245-3715

Keywords: Immunogenicity; Anti-Drug Antibodies; Product Information; Summary of Product Characteristics; Therapeutic Biologics; Recombinant drugs; Biosimilars; European Market

Number of Words: 5150-5200 


\begin{abstract}
Objective: To evaluate whether the current case-by-case practice leads to sufficient reporting of immunogenicity-related information in the Summary of Product Characteristics (SmPCs) of biological products approved in the European market.

Methods: Immunogenicity-related information was identified and extracted from a group of 73 biological drugs that complied with drug-selection criteria. Afterwards, thirteen dichotomous questions were proposed to evaluate whether any issues are being commonly neglected.
\end{abstract}

Results: Most SmPCs (92\%) do not have any recommendations on how to report immunogenicity-related adverse drug reactions by patients or healthcare professionals. Furthermore, $80 \%$ of SmPCs do not-identify the assay used to assess the reported immunogenicity rates, while $81 \%$ do not address the possible impact of immunogenicity on their drug's pharmacokinetics. It was also identified that a group factor (i.e. older drugs' SmPCs) could be influencing how/which issues were being addressed by newerdrugs SmPCs. To transform SmPCs into useful tools when an immunogenic response occurs, a proposal on how to report immunogenicity-related information in the SmPCs of biological products is advanced. This tool can be used by patients, healthcare professionals, regulators and businesses to evaluate the information of SmPCs.

Conclusions: Based on these results, a case-by-case strategy does not yield enough reporting across products and thus immunogenicity-related information should be reported in a systematic way. Further guidance about reporting immunogenicity-related information is required, else SmPCs will not be the basis of information for healthcare professionals on how to use a biological product safely and effectively. 


\section{INTRODUCTION}

Unlike most small-molecules, biological drugs have the inherent possibility to trigger unwanted immunogenic responses against themselves, becoming an additional problem when analyzing their risk-benefit ratio. This hurdle occurs when foreignness or stress signals are perceived by the immune system[1], which responds by developing specific Anti-Drug Antibodies (ADAs). The clinical relevance of these ADAs is often unpredictable and historically it has ranged between no clinical relevance being detected up to life-threatening responses, with other consequences in-between these two extremes[2,3]. To address this problem, European guidelines require the assessment of the immunogenic characteristics of new products for a successful Marketing Authorisation Application (MAA)[4].

Considering that immunogenicity was one of the clinical issues that most frequently caused the rejection of MAAs, because it was not sufficiently analyzed[5], the European Medicines Agency (EMA) has since published several guidelines about assessing and monitoring unwanted immunogenicity[4, 6, 7]. This guidance identifies the plethora of factors $[4,8]$ (disease-, patient- and product-related) possibly promoting immunogenicity, the validation of assays to measure ADA development and how to evaluate immunogenicity during clinical development. Furthermore, it establishes that the thoroughness of an analysis should be based on a risk-assessment strategy so that a deeper analysis is required for higher risk products[9]. Therefore, guidance on the data necessary to assess the immunogenicity of a new biological product is well-established [10]. Furthermore, a growing consensus between the stakeholders has been achieved in recent years, benefitting the medical community by faster and methodical evaluations of MAAs[11]. Despite these advancements, it is still unclear about which data should be included in the Summary of Product Characteristics (SmPC) and how should this information be reported. To solve this issue, a case-by-case analysis is currently performed but the quality of this approach is questionable[11]. This is problematic since SmPCs are one of the tools most commonly used by Healthcare Professionals[12] (HCPs) and since, according to European Guidelines, the SmPC should be "the basis of information for healthcare professionals on how to use the medicinal product safely and effectively"[13].

The objective of the current analysis is to scrutinize whether the current practice leads to sufficient reporting of immunogenicity-related information in the SmPCs of biological 
products approved in the European market, thus raising a discussion on whether SmPCs are currently capable of guiding HCPs in the clinical practice and of promoting informed medical decisions[11, 14, 15].

\section{METHODS}

\subsection{Database of biological drugs}

A list of all the biologic drugs approved by EMA until 2011 (inclusively) was compiled. Drugs approved after 2011 were excluded because newer drugs were expected to have less information about their immunogenicity reported in literature. Furthermore, this analysis investigates whether SmPCs had been updated after receiving a Marketing Authorisation (MA), which would not be a fair evaluation in drugs that have not been extensively used in real-world practice.

Published lists[16-19] were used to identify the biological drugs that were approved by either the Food and Drug Administration (FDA) and/or EMA. Concomitantly, an examination of all the titles obtained from searching recombinant DNA (rDNA) (701 results) and Biosimilar (filter:EPAR) (192 results) in EMA's search engine was performed. Each product identified through these sources was verified in EMA's website to confirm their current approval status.

\subsection{Selection criteria}

Selection criteria were defined to select the drugs that had a higher likelihood of including immunogenicity data in their SmPCs. The rationale for each criterion can be found in Table S1. Briefly, the following criteria were used:

Exclusion criteria:

a. Exclusive indication(s) in Oncology, Rejection of transplants, Myocardial Infarction/Thromboembolism, Infertility;

b. Approval date later than 2011; 
Inclusion criteria:

a. Biotechnological medicines;

b. Biosimilars of a.;

c. MA approved by EMA;

An article from GaBI Online ${ }^{1}$ was also used to pinpoint any unidentified biosimilars. A classification previously published by others[20] was used to categorize each drug in our list into different groups. This classification was proposed considering the categorizations given through the international nonproprietary names (INNs) and/or anatomical therapeutic chemical (ATC).

\subsection{Identification \& extraction of immunogenicity-related information from a SmPC}

A collection of all the immunogenicity-related information detected inside the SmPCs was performed. To identify this data, a search inside each document for the keywords "antibodies/antibody", "neutralizing", "inhibitors", "immunogenicity", "batch” \& "traceability" was performed. If this content had information relevant to the immunogenicity of the drug, it was extracted and compiled in an Excel sheet. The same keywords were used to identify the post-marketing alterations, accessible through the documents available in EMA's website as "Procedural steps taken and scientific information after the authorisation"

\subsection{Analysis of the immunogenicity-related information inside a SmPC}

Based on the previously extracted information from SmPCs, dichotomous questions were devised to evaluate these documents on the most commonly reported and relevant issues related to immunogenicity. Table 1 contains these questions, their relevance and the elements that we were expecting to find in order to classify a SmPC

\footnotetext{
$1 *$ http://www.gabionline.net/Biosimilars/General/Biosimilars-approved-in-Europe
} 
as positively addressing each question. Idiosyncratic situations, that still raised doubts

as to whether a SmPC positively addressed a specific question, were discussed with an

independent researcher and a consensus was reached for each situation.

Table 1: Questions and rationale used to evaluate which issues are addressed in each $\mathrm{SmPC}$

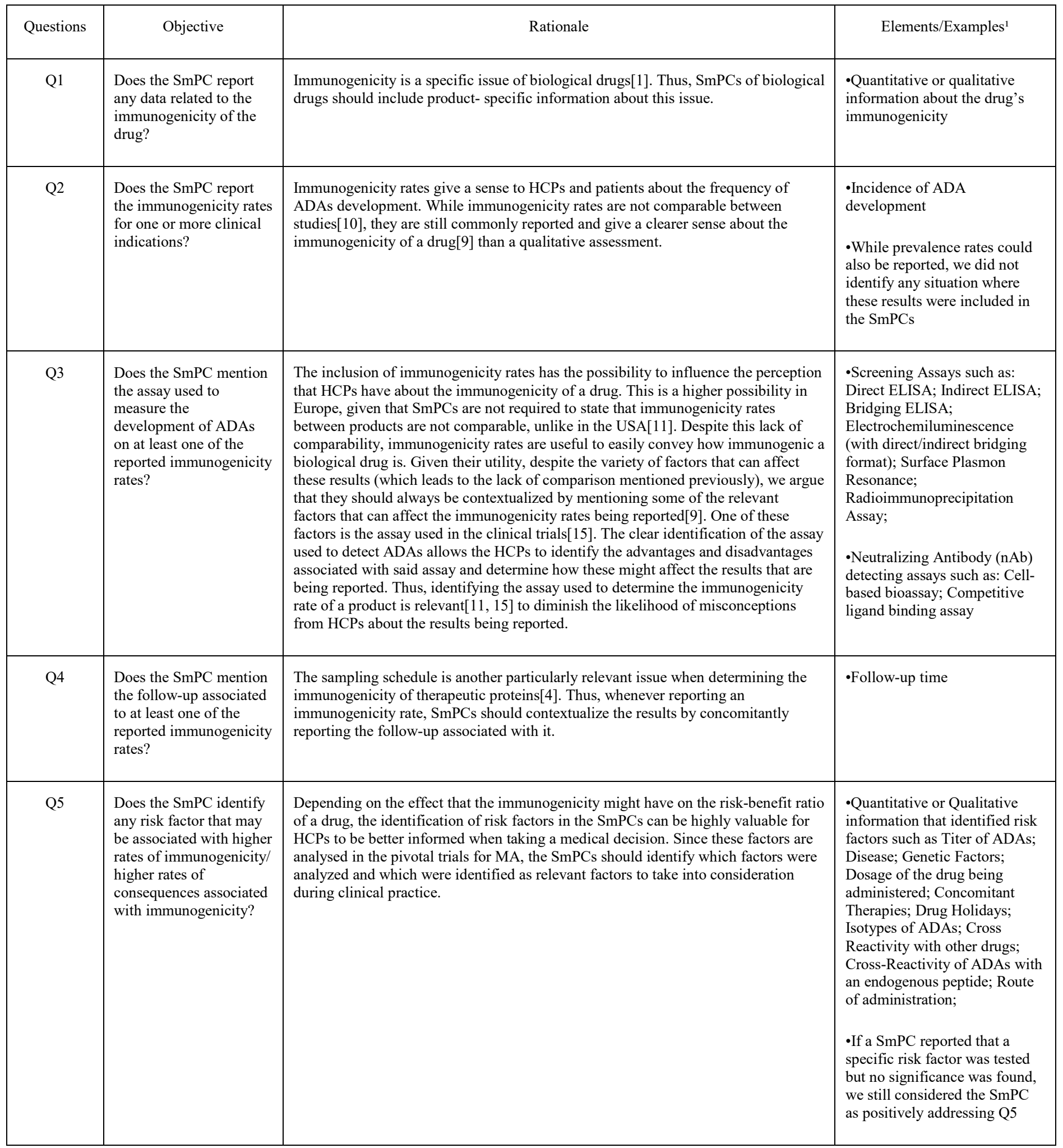




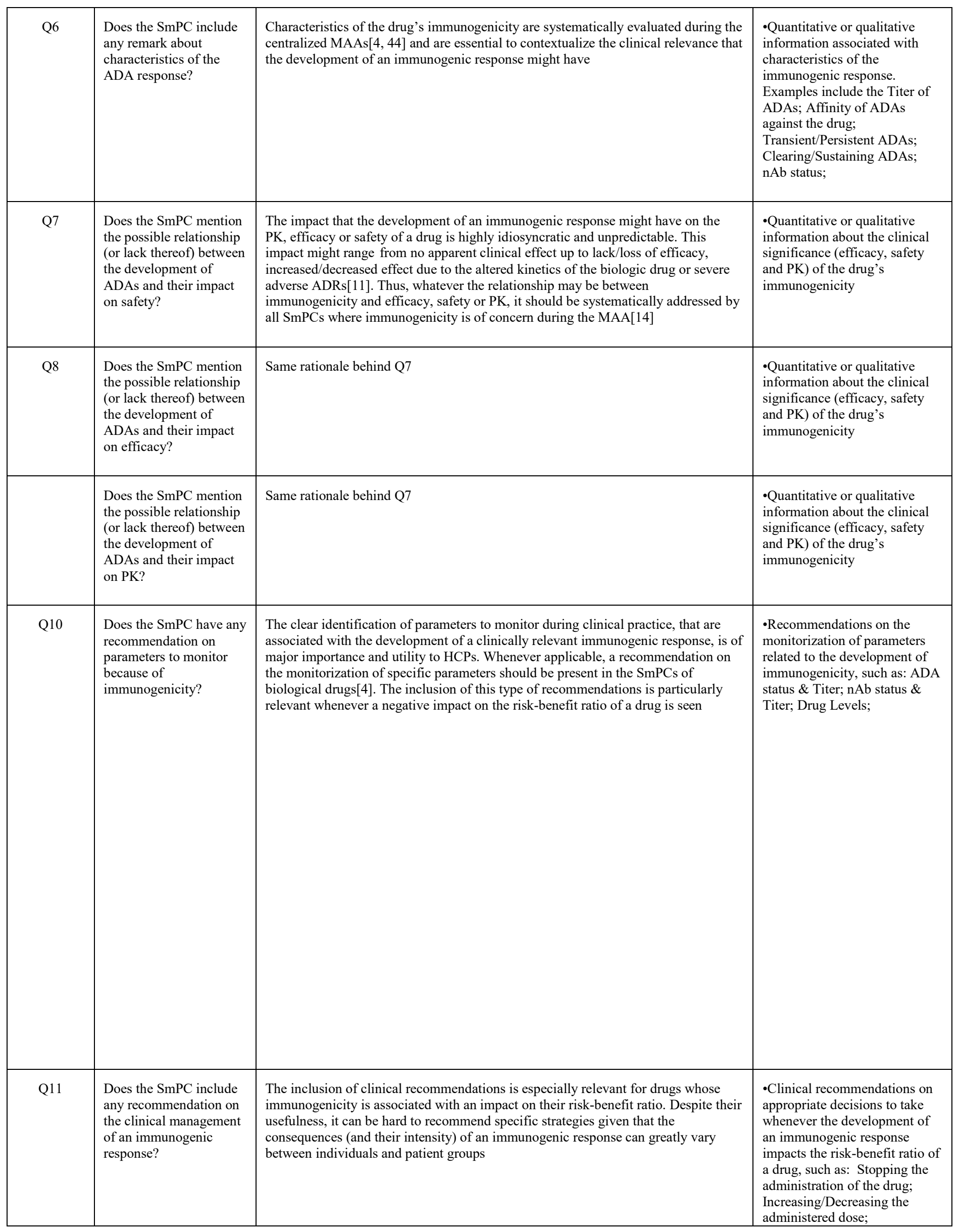




\begin{tabular}{|c|c|c|c|}
\hline & & & $\begin{array}{l}\text { Administration of other support } \\
\text { drugs; }\end{array}$ \\
\hline Q12 & $\begin{array}{l}\text { Does the SmPC } \\
\text { recommend the reporting } \\
\text { of cases (by HCPs or } \\
\text { patients) where } \\
\text { immunogenicity is } \\
\text { detected/ suspected? }\end{array}$ & $\begin{array}{l}\text { The knowledge gathered during the pre-marketing phase about a drug's } \\
\text { immunogenicity is limited[15], given the inherent limitations of these trials[4,45] } \\
\text { (Limited number of patients; Limited external validity) to detect long term and/or } \\
\text { rare effects. Furthermore, the introduction of manufacturing changes can have an } \\
\text { unforeseen impact on the immunogenicity of a drug. Thus, a solid } \\
\text { pharmacovigilance system must be in place for HCPs and patients to keep trust in } \\
\text { these products. This is reflected in the various guidelines related to } \\
\text { pharmacovigilance or risk management plans. Given the relevance and utility of the } \\
\text { pharmacovigilance system in keeping high standards of safety and efficacy, SmPCs } \\
\text { should clarify when a report related to the development of immunogenicity is } \\
\text { justified and what additional information is relevant to be included in this report. }\end{array}$ & $\begin{array}{l}\text {-Identification of situations } \\
\text { where a report by the HCPs or } \\
\text { patients should be performed. } \\
\text { These situations include: The } \\
\text { confirmation of an ADA } \\
\text { positive status; The } \\
\text { development of clinical } \\
\text { consequences (with an impact } \\
\text { on safety or efficacy) } \\
\text { previously associated with an } \\
\text { immunogenic response; }\end{array}$ \\
\hline Q13 & $\begin{array}{l}\text { Has the SmPC been } \\
\text { updated with new } \\
\text { information related to } \\
\text { immunogenicity after the } \\
\text { initial MA? }\end{array}$ & $\begin{array}{l}\text { SmPCs are dynamic documents that must be updated throughout a products' market } \\
\text { life. As previously mentioned, the knowledge about a drug's immunogenicity is } \\
\text { limited before its use in the real world and further knowledge is gained and } \\
\text { published as the experience with these drugs increases. SmPCs should be expected } \\
\text { to reflect the knowledge that is obtained and accepted by the medical and academic } \\
\text { communities, thus increasingly requiring updates as their real-world experience } \\
\text { increases }\end{array}$ & $\begin{array}{l}\text {-Identification of updates } \\
\text { (reported in EMA's documents } \\
\text { "Procedural steps taken and } \\
\text { scientific information after the } \\
\text { authorisation", specific for each } \\
\text { drug) related to } \\
\text { immunogenicity. Updates that } \\
\text { were merely including } \\
\text { immunogenicity data necessary } \\
\text { for a new clinical indication } \\
\text { were not considered as positive } \\
\text { situations; }\end{array}$ \\
\hline
\end{tabular}

1- The examples are non-exclusive and represent the expected answers/information for each question. A caseby-case analysis was performed for each SmPC considering the context given in each document. Situations that did not fall under the scope of these examples were heavily discussed with an independent analyst until a conclusion was reached.

\section{RESULTS}

A list of all the products that complied with our criteria had to be created. Afterwards, a classification proposed by other authors[20] was used to categorize these drugs into larger groups. The full list can be accessed in Table S2.

The motivation behind these exclusion and inclusion criteria was to select the drugs with the highest potential of developing unwanted immunogenicity since these drugs are expected to have a high degree of information in their SmPC regarding their immunogenicity and its relationship with the risk-benefit ratio. Figure S1 shows that the majority (37/73) of the list is composed of Monoclonal Antibodies (mAbs), Insulins and Enzymes. Other groups that also consist of a considerable number of drugs are the Interferons, the Antihaemophilic Factors and the Epoetins. The full list is available in Table S2.

Despite having been identified and compiled into our list, Biosimilars are not included in further analyses. A dedicated section to the issue of Biosimilars and their SmPCs is presented in the discussion. 
To analyze the immunogenicity data in the SmPC of each drug, a group of dichotomous questions was created. This 'questionnaire' can be seen in Table 1 and attempts to reflect the core immunogenicity-related topics that are addressed by these documents. These questions can be further divided into two groups: Group 1 consists of questions Q1-Q6 and its focus is on the degree of detail that is conveyed by the SmPCs about the risk of development of ADAs. The second group is composed of questions Q7-Q12 and its focus is on the information conveyed by the SmPCs regarding the clinical impact (and its management) of immunogenicity. Question Q13 does not fit any of these two groups since it measures whether any new information about immunogenicity has been updated into the SmPCs.

The focus of our analysis is not on the specific content presented in each SmPC, since such an analysis is out of the scope of this article. Instead, this evaluation is designed to identify which issues related to immunogenicity are not being addressed by the SmPCs, thus hindering the SmPCs of being the basis of information to HCPs. Given the inherent heterogeneity that exists across the SmPCs, the framing of each question was intended to be broad. Additionally, our decision-making process was based on a tolerant mindset, in which the bare minimum information about each question was enough to classify a SmPC as positively addressing the question. This permits the estimation of the maximum number of SmPCs that address these issues by excluding false negatives.

\subsection{Evaluation of SmPCs}

Figure 1 shows the number of SmPCs that address each of the questions mentioned previously. To give a sense of proportion, the first column shows the total number of SmPCs that were analyzed. A clear distinction can be identified between the relevance across SmPCs that some topics have compared to others.

Looking at the first group of questions, 37\% (27/73) do not report any immunogenicity rates and thus this risk is not numerically quantified in these SmPCs. Since Q3 and Q4 only make sense to be addressed when the immunogenicity rates are reported in the SmPCs, a drop (particularly intense for Q3) is seen. Q3 is related to the methodology that was used to detect the ADAs and only $20 \%(9 / 46)$, out of the documents that report immunogenicity rates, concomitantly report the assay used to measure the development 
of ADAs. A substantially higher proportion (61\% [28/46]) of SmPCs concomitantly report the follow-up associated with at least one immunogenicity rate.

Looking at Q5 and Q6, we see that about half of SmPCs (45\% [33/73] and 52\% (38/73]) address these issues by respectively giving some complementary information related to risk factors and characteristics of the immunogenic responses that were identified or analyzed.

About the second group of questions (Q7-Q12), the majority of the SmPCs (70\% [52/75) for Q7 \& 67\% [50/75] for Q8) contemplate the relationship (or lack thereof) that the development of immunogenicity may respectively have with the safety and efficacy of these drugs. On the other hand, sharply contrasting with the previous questions, $81 \%$ (59/73) of all SmPCs neglect to address Q9 by not mentioning the relationship (or lack thereof) between immunogenicity and the PK of these drugs.

Regarding the proportion of SmPCs issuing recommendations to address Q10, only $33 \%(24 / 73)$ of all SmPCs give any guidance to HCPs and patients, leaving the remaining SmPCs without any clarifications about the clinical circumstances that may justify monitoring the immunogenicity status of a patient or the drug's plasma levels. Q11 complements the previous question by analyzing if there are any recommendations to minimize the impact of immunogenicity on safety or efficacy. The same proportion of SmPCs as in Q10 was detected. As recognized in Table 1, Q10 and Q11 are questions that may not make sense to be addressed whenever a lack of relevance or knowledge about immunogenicity is detected. Since our analysis includes SmPCs in both situations, the proportion of SmPCs that are expected to address these issues is probably being underestimated.

A strong medical culture on reporting Adverse Drug Reactions (ADRs) whenever these occur is particularly important in biological drugs, given the impact that manufacturing changes might have on the risk-benefit ratio (through altered immunogenicity, for example) of a drug[21,22]. Furthermore, given that uncertainty about the clinical relevance of immunogenicity of a biological product is a common problem after the $\operatorname{MA}[9,15]$, it further increases the relevance of pharmacovigilance. Q12 evaluates the proportion of SmPCs that advise the HCPs or patients to report immunogenicitypositive cases (i.e. ADA positive patients) or cases where consequences related to 
immunogenicity are suspected. Only 8\% (6/73) of all SmPCs included recommendations of this kind, potentially hindering the perception the patients and the medical community about the relevance of assessing and reporting these situations. Finally, approximately 43\% (30/70) of the SmPCs have had at least one post-marketing change to their SmPC that was associated with an update on immunogenicity data. Three drugs are excluded from this last analysis because they were approved nationally (Genotropin, Eprex and Neupogen). A justification for these exceptions be found in Table S1.

Out of all the issues analyzed, Q12 was the least addressed issue, followed by Q3 and Q9, respectively.

Figure 2 further divides the positive answers according to the previously mentioned classification. This analysis shows a few trends that are possibly relevant to how immunogenicity results are being reported by the SmPCs: 1) In Q2, almost none of the SmPCs from Epoetins and Insulins reported any immunogenicity rates, which is peculiar given that the other groups address this issue in large proportions. Q4 and Q8 are other examples of similar situations. 2) The relationship between ADAs and the Pharmacokinetics (PK) (Q9) is only addressed by mAbs and Enzymes, which was not expected given the relevance that this relationship has for biotechnological drugs in general[11].

Overall, these trends lead us to suspect that previously published SmPCs could be influencing how the information is presented and which topics are addressed in the SmPCs of newer drugs from the same group, meaning that a group factor can be influencing how information about new drugs is being presented. While other factors (of which we are unaware) could explain these trends, further trends increase the likelihood of this hypothesis:

1) A very small proportion of SmPCs mention that comparisons between the immunogenicity of different products is not appropriate. When identifying these drugs, we noticed that only abatacept, certolizumab Pegol, golimumab, adalimumab, Rebif and Avonex include this statement. These drugs are either mAbs (All of them are Anti TNF$\alpha$ drugs except for abatacept, which has nonetheless the same indications) or 
Interferons-1b. The fact that this specific information is included in the SmPCs of drugs with the same indications appears to support our hypothesis.

2) Looking at the SmPCs of all Human Insulins, it becomes clear that the information about immunogenicity reported in all of them (excluding Insuman) is exactly the same across products. In all of these products, it is only mentioned that "No profound binding to plasma proteins, except circulating insulin antibodies (if present) has been observed." [23-27]. This observation of equal information across SmPCs was also identified in Epoetins and Antihaemophilic Factors. A possible reason is that these drugs are commercially explored by the same MAH, which is true for most but not all products.

\section{DISCUSSION}

Two points of contention arise from this investigation. First point is about the selected issues to evaluate the SmPCs. To reflect the "state of the art" on reporting immunogenicity data in SmPCs, all information about immunogenicity was collected before specifying any questions, allowing us to understand which issues were most commonly reported. This strategy explains why most questions are positively answered by $25 \%$ or more of the SmPCs analyzed. The second problem arises from evaluating whether a SmPC positively addresses each question, given its subjectivity. To mitigate this problem, several strategies were used. Firstly, the decision of whether a SmPC answered positively to each question was always performed by the same person. Secondly, expected elements for each question were identified a priori. Thirdly, discussions with an independent reviewer were performed for idiosyncratic situations.

This analysis confirms the need to create a common framework for reporting immunogenicity-related issues with respect to biologicals that should be addressed in SmPCs. Several cases were identified where the data being reported was insufficient to inform the HCPs about the inherent immunogenicity of these biologicals. In other cases, data about immunogenicity was reported but not enough additional information of relevance was given to contextualize these results, creating the potential for misconceptions in the medical community about the immunogenicity of a specific product[11]. Furthermore, several signs suggest that a group-factor could be influencing how information is being reported in some SmPCs. 
Overall, we argue that these circumstances arise from a lack of guidance about reporting immunogenicity data. The current European guidelines clearly identify the requirements for immunogenicity assessment that must be fulfilled in the Common Technical Document (CTD) for a successful MAA[4, 14]. Furthermore, recently revised guidelines about Pharmacovigilance[7] issue more guidance about immunogenicity. Notwithstanding, very few clarifications are given about the data that is expected to be reported in SmPCs. Therefore, while clear and specific guidance exists about the necessary data for a successful MAA, the same cannot be said about which of this data should be transcribed into the SmPCs to inform HCPs and patients about the immunogenic potential of biological products. Considering that the immunogenicity of a drug has the potential to greatly affect the risk-benefit ratio of said medicine, not reporting/inconsistently reporting immunogenicity-related information hinders the capability of SmPCs to be "the basis of information for healthcare professionals on how to use the medicinal product safely and effectively"[13].

Regarding our analysis, the first matter worth considering regards which of these questions should be addressed systematically. Our view is that each approved clinical indication should be associated with immunogenicity-related information from the pivotal clinical trials necessary to obtain the MA for said indication. This information should be sufficient to address Q2-Q9 if no clinically relevant impact has been verified in that clinical setting. However, if a relevant impact was detected, efforts should be pursued to also address Q10 and Q11. If Q2-Q9 have already been previously addressed and new data about immunogenicity is being added to update the SmPCs, we argue that at least Q2-Q4 should be addressed. When addressing these issues (especially those from the second groups of questions), the depth of analysis presented in the SmPCs should reflect the possible impact of the development of ADAs, with more information being expected when an impact is verified, thus mimicking the current risk-based analysis for drug development[14]. A recommendation on reporting ADRs related to immunogenicity should also be present once in every SmPC to promote a robust culture of pharmacovigilance. A parallel issue worth mentioning is the traceability of biological products. Current guidelines already recommend SmPCs to state that " $(. .$.$) the name and$ batch number of the administered product should be clearly recorded"'[7]. Our analysis only identified 20 out of 70 centrally approved products that included this statement in 
their SmPCs (data not shown) meaning that systematically including this recommendation is also desirable.

Figure 3 reflects our view on which questions should be addressed by the SmPCs. This tool allows HCPs, patients, regulators and MAHs to evaluate the immunogenicity data in a specific SmPC and conclude whether the information being presented addresses the most common issues related to immunogenicity or not. A potential problem with our proposal is that SmPCs should not address issues for which data is lacking[28]. However, several SmPCs were detected with a statement acknowledging the lack of data about immunogenicity for specific situations. Therefore, given the possible impact of immunogenicity on the risk-benefit ratio of biological drugs, we argue that a lack of knowledge for one (or more) of these issues is relevant information to be communicated and that these are the ideal situations to redirect HCPs and patients to the RiskManagement Plan (RMP) and European Public Assessment Report (EPAR) of the product for more information.

The second matter worth discussing arises from the least addressed questions. Q12, Q3 and Q9 were unaddressed by $80 \%$ or more of the SmPCs that could have addressed these issues, which is from our point of view a lost opportunity to clarify HCPs and patients.

Regarding Q12, we were surprised to find that most SmPCs are not currently being used as a tool to promote the practice of reporting ADRs. The ADRs possibly related to the occurrence of an immunogenic response are especially relevant to be reported since this is one of the probable mechanisms behind the occurrence of new ADRs after manufacturing changes. Furthermore, if a causal link between an ADR and the occurrence of an immunogenic response is suspected/confirmed, other factors not usually asked to be reported could be relevant and the SmPC should clarify these aspects. We defend a pragmatic approach, where the reporter is expected to identify the $\mathrm{ADA}$ and $\mathrm{nAb}$ status of the patient (if determined), risk factors possibly associated with the occurrence of the immunogenic response and the cumulative exposure time. Other issues can possibly be relevant to report and should be considered in a case-by-case basis. While our analysis does not focus on other tools besides the SmPC, it's worth 
mentioning that current guidelines recommend including immunogenicity in the RMP of recently approved medicines and that other pharmacovigilance tools can be further used as risk minimization strategies[15], overall contributing to the consolidation of a strong and reliable pharmacovigilance system for biological drugs.

Regarding Q3, immunogenicity rates are not comparable between studies. However, they provide useful estimates of how frequently immunogenicity occurs during real world usage, thus having the potential to influence HCPs on their perception of the immunogenic potential of a new biological medicine. One of the factors particularly relevant to consider when developing an immunogenicity assessment plan is the assay that will be used to measure the ADA status of the patients[9] and this factor alone can have a great impact on the immunogenicity rates of a product[29, 30]. We argue that not systematically including a recommendation about the inappropriateness of comparing immunogenicity rates from different studies in addition to not identifying the methodology used to measure the immunogenicity rates reported in the SmPCs has the potential to induce HCPs into relevant misunderstandings regarding the immunogenicity of biological products. Therefore, corrective measures are necessary to inform HCPs in a clear and transparent manner. A similar statement about the inappropriateness of comparing cross-product immunogenicity rates is already required in every product information by the FDA[11].

Another issue that our analysis detected is that the large majority of the SmPCs did not clarify whether the development of ADAs had an impact on the PK of these drugs. Nonneutralizing antibodies may affect the risk-benefit ratio of a drug by increasing (clearing ADAs) or decreasing (sustaining ADAs) a medicine's clearance rate[10, 31], possibly leading to a lack of efficacy or to an increase in frequency/severity of ADRs. In both cases, the impact surges because ADAs promote the drug's concentrations to be outside of the therapeutic window. Given this possibility, CTDs need to contain data about this relationship for a successful MAA[32] but the same standard in not applied in the SmPCs of biological drugs. Neglecting to report this information is, in our opinion, undermining the assessment of the HCPs regarding the impact of an immunogenic response.

Curiously, a previous study that analyzed the prescribing information of 121 biological drugs approved by FDA[33] reports equivalent proportions to our study regarding the 
drugs that address the clinical impact of immunogenicity on safety $(60 \%)$, efficacy (49\%) and PK (26\%). Despite not evaluating other issues, this comparability across studies leads us to suspect that the same issues identified here might also be applicable to other regulatory regions. A further analysis on these different regions is therefore necessary.

The third matter from our analysis worth considering is related to Q13. 43\% of the SmPCs analyzed had at least one post-marketing change associated with immunogenicity. Therefore, this obviously implies that $57 \%$ of the biologic drugs that have been authorised prior to 2012 did not have their SmPC updated not even once when it comes to data related to immunogenicity. Being a field in rapid development, especially regarding the methodologies used to detect ADAs[10], and considering that immunogenicity is required to be included in the RMP of biological drugs, we were expecting that a much larger proportion of these older SmPCs (MA prior to 2011) had been updated. Therefore, one could ask whether new information about these drugs has been published and accepted by the scientific and medical communities which could have deserved an update of these SmPCs. While our analysis does not answer the previous question, it's undeniable that the immunogenicity rates of some SmPCs do not currently reflect the immunogenicity rates being published with newer and more sensitive assays. Given that these rates influence the perception of the medical community about how often the development of an immunogenic response against a biological drug occurs, we wonder if the SmPCs of older drugs should not be updated to also reflect the immunogenicity rates that are being detected with these newer assays instead of just reporting results based on assays developed 10-15 years ago. A perfect opportunity to update the information in some of these SmPCs arises from the approval of biosimilars.

Having made our case for the value of systematic reporting in informing prescribers, we concede that this may have relatively little influence on clinical management decisions particularly with respect to use of biologicals that have been in clinical use for a long time. This is large part because of the idiosyncratic nature of the clinical relevance of measurement of ADA. However, this information might become more relevant if data were made available regarding the proportion of ADA that are neutralizing versus the proportion of non-neutralizing antibodies. This would assume practical relevance in the 
context of clinical practice which includes measurement of trough levels of administered biologic and might have implications for dose optimization strategies.

\subsection{Biosimilars' SmPCs and immunogenicity data}

According to European guidelines, biosimilars are medical products comprised of an active substance from an already established drug (reference product) in the EEA. These biosimilar products must have proven a high degree of similarity (regarding their quality, biological and medical properties) between themselves and their reference products[7].

The Biosimilar Medicinal Products Working Party (BMWP) reported in 2012 three different possibilities regarding the inclusion of bioequivalence data into the SmPC of biosimilar drugs. These possibilities ranged from not including any information at all up to only reporting the bioequivalence data supporting EMA's assessment[34]. Current practice about a Biosimilar's SmPC in Europe is to develop a document identical (with the exception of using the INN of the active substance instead of the tradename of the reference product[35]) to the $\mathrm{SmPC}$ of the reference product, thus excluding any bioequivalence data.

Head to head studies comparing a biosimilar to its reference counterpart are a regulatory requirement. The low predictability that non-clinical models confer regarding the unwanted immunogenicity of biological drugs is one of the issues that contributes to the requirement of clinical studies for bioequivalence. Therefore, immunogenicity data is an important issue to analyze when determining the biosimilarity between two products. Furthermore, other issues possibly related to immunogenicity such as switching and extrapolation of indications are commonly raised by the scientific and medical communities regarding the use of biosimilars. While current practice is to include the details of the bioequivalence studies exclusively in the EPARs developed by EMA[35], we argue that this is an inadequate strategy given that low proportions of HCPs use EPARs as a source of information[12]. Furthermore, not including enough information about the bioequivalence studies in the SmPCs increases the likelihood of misunderstandings and unreasonable fears by some members of the medical community. On the other hand, the regulators' reasoning that different SmPCs between 
products with a similar active substance could lead to misunderstandings is also sensible [31]. Therefore, we support the idea that both the SmPCs of the reference product and of the biosimilar product should be updated to include data from the bioequivalence studies, specifically the data related to the immunogenicity of both products.

From the reference products' perspective, we argue that this update is necessary given that most of these drugs have been on the market for over 10 years. During this period, an increasing number of improvements regarding the assessment of immunogenicity have occurred but many SmPCs include data mostly obtained during the drug's pivotal clinical trials[11]. Consequently, the immunogenicity rates reported by these documents are possibly underestimated in some drugs' SmPCs[1]. Our reasoning is that including the data from bioequivalence studies, assessed with new methodologies, could be an excellent opportunity to update these drugs' SmPCs thus providing a better sense of amplitude on the detected incidence of ADAs between studies. Moreover, including immunogenicity rates from the bioequivalence studies would almost surely require a concomitant statement about the incomparability between immunogenicity rates from different studies, which we also see as a positive outcome.

From the biosimilars' perspective, we argue that this update is necessary because several issues, including immunogenicity[36] but also switching/interchangeability[37, 38 ] and extrapolation of indications[31] have been raised throughout the years. Including immunogenicity data collected during the bioequivalence studies can help clarify some of these issues in a document that is regularly used by HCPs. Additionally, reporting data that supports the decision to approve a biosimilar into the European Market might help invalidate the perception that the evidence supporting this decision is insufficient[31], while excluding data from bioequivalence studies foments this perception.

Common updates to SmPCs of different biological products which contain the same or closely related active substance can be seen in several situations and examples of these include the updates A31/0134, A31/0078 and A31/0178 about the Sippet study for ReFacto AF, Advate and Helixate NexGen respectively[39-41] or the update IB/0002/G for both Biopoin and Eporatio[42, 43]. 
Applying the same principles, an update should also be required whenever a bioequivalence study between the pre and post-change versions of a product in humans is necessary. The arguments just mentioned for biosimilars are also applicable in these cases and we also think that including this data in the SmPCs of biological products raises awareness in the medical community about manufacturing changes that can have an impact on the risk-benefit ratio of the altered drug, further complementing the increased vigilance that is required after a change is proposed and approved[7].

\section{CONCLUSIONS}

This analysis focuses on the absence of a common set of problems related to immunogenicity that should be addressed by a drug's SmPC whenever said drug has the potential to develop clinically relevant ADAs. Currently, each SmPC reports the information that is considered as the most pertinent during the MAA but this practice leads to a great degree of heterogeneity between SmPCs.

Given the clinical relevance that the development of ADAs might have, we argue that more guidance on how to report immunogenicity data is necessary. Until then, SmPCs of biological drugs will not be accomplishing their function as the basis of information for HCPs on how to use the medicinal product safely and effectively because a potentially impactful issue on the efficacy and safety of these products is not being adequately addressed by a large proportion of SmPCs. To contribute with solutions to this problem, a set of common issues necessary to be addressed is proposed. We hope that this tool can increase the quality and transparency of the information being reported in the SmPCs, thus promoting informed clinical decisions that increase the quality of care.

A specific analysis about biosimilars' SmPCs is also included, given their relevance in the current and future medical practice. Nowadays, SmPCs of biosimilar drugs are a complete reflection of the SmPCs of the reference product. We consider this practice to be unreliable given the relevance that some data from bioequivalence studies can have on clarifying some of the major issues that have been raised ever since biosimilars were firstly approved into the European market. We argue that introducing bioequivalence data into the SmPCs of the reference product and of the biosimilars that have been approved by EMA is a better alternative, with benefits to both products. The same 
strategy should also be applied to manufacturing changes that required bioequivalence studies in humans to be performed.

Despite this analysis and the several proposals recommended here, the main purpose of this study was to diagnose a problem of communication caused by a lack of insufficient immunogenicity-related information in the SmPCs of biological drugs. We believe that addressing the issues raised in this analysis is responsibility of the regulatory agencies and therefore urge regulatory clarification about these issues by including more guidance in future European guidelines or other appropriate sources. Guidance about reporting immunogenicity data on biological drugs' SmPCs will become ever more relevant given that increasingly greater numbers of biological products (including biosimilars) will be applying for MAs to the European Market.

\section{Author Contributions}

\section{$7 \quad$ Acknowledgments}

We express our gratitude to Carlos Araújo for providing his time to evaluate, together with RB, SmPCs whose appraisal was not certain to RB.

\section{Conflict of Interest}

$\mathrm{RB}$ declares no commercial or financial relationship that could be construed as a potential conflict of interest.

\section{$9 \quad$ Funding}

No funding was received for this manuscript.

\section{REFERENCES}

1. Gunn GR, Sealey DCF, Jamali F, et al. From the bench to clinical practice: understanding the challenges and uncertainties in immunogenicity testing for biopharmaceuticals. Clin Exp Immunol. 2016;184(2):137-46.

2. Schellekens H. Immunogenicity of Therapeutic Proteins : Clinical Implications and Future Prospects. Clin Ther. 2002;24(11):1720-40.

3. Kuus-reichel K, Grauer LS, Karavodin LM, et al. Will Immunogenicity Limit the Use, Efficacy, and Future Development of Therapeutic Monoclonal Antibodies? Clin Diagn Lab Immunol. 1994;1(4):365-72. 
4. European Medicines Agency. EMEA/CHMP/BMWP/14327/2006- Guideline on Immunogenicity assessment of therapeutic proteins. Available at: https://www.ema.europa.eu/en/documents/scientific-guideline/guidelineimmunogenicity-assessment-therapeutic-proteins-revision-1_en.pdf [Accessed March 2019]

5. Schneider CK, Dallman GS. Typical pitfalls in applications for marketing authorization of biotechnological products in Europe. Nat Rev Drug Discov. 2008;7(11):893-9.

6. European Medicines Agency. EMEA/CHMP/BMWP/101695/2006- Guideline on Comparability after a change in the Manufacturing Process- Non-Clinical and Clinical Issues. Available at:https://www.ema.europa.eu/en/documents/scientificguideline/guideline-comparability-biotechnology-derived-medicinal-productsafter-change-manufacturing-process_en.pdf [Accessed March 2019]

7. European Medicines Agency. EMA/168402/2014- Guideline on good pharmacovigilance practices (GVP) Product- or Population-Specific Considerations II: Biological medicinal products. Available at: https:/www.ema.europa.eu/en/documents/scientific-guideline/guideline-goodpharmacovigilance-practices-gvp-product-population-specific-considerationsii_en-0.pdf [Accessed March 2019]

8. Ponce R, Abad L, Amaravadi L, et al. Immunogenicity of biologically-derived therapeutics: Assessment and interpretation of nonclinical safety studies. Regul Toxicol Pharmacol. 2009;54(2):164-82.

9. Shankar G, Pendley C, Stein KE. A risk-based bioanalytical strategy for the assessment of antibody immune responses against biological drugs. Nat Biotechnol. 2007;25(5):555-61.

10. Chirmule N, Jawa V, Meibohm B. Immunogenicity to Therapeutic Proteins : Impact on PK / PD and Efficacy. AAPS J. 2012;14(2):296-302.

11. Shankar G, Arkin S, Cocea L, et al. Assessment and Reporting of the Clinical Immunogenicity of Therapeutic Proteins and Peptides - Harmonized Terminology and Tactical Recommendations. AAPS J. 2014;16(4):658-73.

12. Dolinar RO, Reilly MS. Biosimilars naming, label transparency and authority of choice - survey findings among European physicians. GABI Journal. 2014;3(2):58-62.

13. European Medicines Agency. A Guideline on Summary of Product Characteristics (SmPC). Available at: https://ec.europa.eu/health/sites/health/files/files/eudralex/vol2/c/smpc_guideline_rev2_en.pdf [Accessed March 2019]

14. Koren E, Smith HW, Shores E, et al. Recommendations on risk-based strategies for detection and characterization of antibodies against biotechnology products. $J$ Immunol Methods. 2008;333(1-2):1-9.

15. Jahn EM, Schneider CK. How to systematically evaluate immunogenicity of 
therapeutic proteins - regulatory considerations. N Biotechnol. 2009;25(5):2806.

16. Usmani SS, Bedi G, Samuel JS, et al. THPdb : Database of FDA-approved peptide and protein therapeutics. PLoS One. 2017;12(7):1-12.

17. Baker MP, Reynolds HM, Lumicisi B, et al. Immunogenicity of protein therapeutics - The key causes, consequences and challenges. Self Nonself. 2010;1(4):314-22.

18. Gorzelany JA, Souza MP. Protein Replacement Therapies for Rare Diseases: A Breeze for Regulatory Approval ? Sci Transl Med. 2013;5(178):1-4.

19. Ecker DM, Jones SD, Levine HL. The therapeutic monoclonal antibody market. MAbs. 2015;7(1):9-14.

20. Klein K, Bruin ML, Broekmans AW, et al. Classification of Recombinant Biologics in the EU: Divergence Between National Pharmacovigilance Centers. BioDrugs. 2015;29(6):373-9.

21. Schellekens H, Casadevall N. Immunogenicity of recombinant human proteins : causes and consequences. J Neurol. 2004;251:4-9.

22. McKoy JM, Stonecash RE, Cournoyer D, et al. Epoetin-associated pure red cell aplasia: past, present, and future considerations. Transfusion. 2009;48(8):175462.

23. European Medicines Agency. Mixtard Summary of Product Characteristics. Available at: https://www.ema.europa.eu/en/documents/productinformation/mixtard-epar-product-information_en.pdf [Accessed March 2019]

24. European Medicines Agency. Protaphane Summary of Product Characteristics. Available at: https://www.ema.europa.eu/en/documents/productinformation/protaphane-epar-product-information_en.pdf [Accessed March 2019]

25. European Medicines Agency. Actraphane Summary of Product Characteristics. Available at: https://www.ema.europa.eu/en/documents/productinformation/actraphane-epar-product-information_en.pdf [Accessed March 2019]

26. European Medicines Agency. Actrapid Summary of Product Characteristics. Available at: https://www.ema.europa.eu/en/documents/productinformation/actrapid-epar-product-information_en.pdf [Accessed March 2019]

27. European Medicines Agency. Insulatard Summary of Product Characteristics. Available at: https:/www.ema.europa.eu/en/documents/productinformation/insulatard-epar-product-information_en.pdf [Accessed March 2019]

28. European Medicines Agency. Summary of product characteristics (SmPC)- What is it and what does it contain? Available at: https:/www.ema.europa.eu/en/documents/presentation/presentation-summaryproduct-characteristics-what-it-what-does-it-contain_en.pdf [Accessed April 2019] 
29. Bloem K, Leeuwen A, Verbeek G, et al. Systematic comparison of drug-tolerant assays for anti-drug antibodies in a cohort of adalimumab-treated rheumatoid arthritis patients. J Immunol Methods. 2015;418:29-38.

30. Hart MH, Vrieze H, Wouters D, et al. Differential effect of drug interference in immunogenicity assays. J Immunol Methods. 2011;372(1-2):196-203.

31. Ebbers HC, Chamberlain P. Controversies in Establishing Biosimilarity: Extrapolation of Indications and Global Labeling Practices. BioDrugs. 2016;30(1):1-8.

32. Sailstad JM, Amaravadi L, Gorovits B, et al. A White Paper - Consensus and Recommendations of a Global Harmonization Team on Assessing the Impact of Immunogenicity on Pharmacokinetic Measurements. AAPS J. 2014;16(3):48898.

33. Wang YMC, Hon YY, Zhou L, et al. Evaluating and Reporting the Immunogenicity Impacts for Biological Products - a Clinical Pharmacology Perspective. AAPS J. 2016;18(2):395-403.

34. European Biopharmaceutical Enterprises. Tell me the whole story: the role of product labelling in building user confidence in biosimilars in Europe. GABI Journal. 2014;3(4):188-92.

35. European Medicines Agency, European Commission. Biosimilars in the EU Information guide for healthcare professionals. Available at: https://www.ema.europa.eu/en/documents/leaflet/biosimilars-eu-informationguide-healthcare-professionals_en.pdf [Accessed April 2019]

36. Belmonte RG, Chirlaque $\mathrm{CH}$, Amador MA, et al. Biosimilars: concepts and controversies. Pharmacol Res. 2018;133:251-64.

37. Cohen HP, Blauvelt A, Rifkin RM, et al. Switching Reference Medicines to Biosimilars : A Systematic Literature Review of Clinical Outcomes. Drugs. 2018;78(4):463-78.

38. Mckinnon RA, Cook M, Liauw W, et al. Biosimilarity and Interchangeability: Principles and Evidence: A Systematic Review. BioDrugs. 2018;32(1):27-52.

39. European Medicines Agency. ReFacto AF- Procedural steps taken and scientific information after the authorisation. Available at: https://www.ema.europa.eu/en/documents/procedural-steps-after/refacto-af-eparprocedural-steps-taken-scientific-information-after-authorisation en.pdf [Accessed March 2019]

40. European Medicines Agency. Advate- Procedural steps taken and scientific information after the authorisation. Available at: https://www.ema.europa.eu/en/documents/procedural-steps-after/advate-eparprocedural-steps-taken-scientific-information-after-authorisation_en.pdf [Accessed March 2019]

41. European Medicines Agency. Helixate NexGen- Procedural steps taken and 
scientific information after the authorisation. Available at:

https:/www.ema.europa.eu/en/documents/procedural-steps-after/helixatenexgen-epar-procedural-steps-taken-scientific-information-afterauthorisation_en.pdf [Accessed March 2019]

42. European Medicines Agency. Biopoin- Procedural steps taken and scientific information after the authorisation. Available at:

https://www.ema.europa.eu/en/documents/procedural-steps-after/biopoin-eparprocedural-steps-taken-scientific-information-after-authorisation en.pdf [Accessed March 2019]

43. European Medicines Agency. Eporatio- Procedural steps taken and scientific information after the authorisation. Available at:

https://www.ema.europa.eu/en/documents/procedural-steps-after/eporatio-eparprocedural-steps-taken-scientific-information-after-authorisation_en.pdf [Accessed March 2019]

44. Rup B, Pallardy M, Sikkema D, et al. Standardizing terms, definitions and concepts for describing and interpreting unwanted immunogenicity of biopharmaceuticals: recommendations of the Innovative Medicines Initiative ABIRISK consortium. Clin Exp Immunol. 2015;181(3):385-400.

45. Rothwell PM. Factors That Can Affect the External Validity of Randomised Controlled Trials. PLoS Clin Trials. 2006;1(1):1-5.

Figure 1: Number of SmPCs positively addressing each question raised in Table 1

Figure 2: Number of SmPCs from each group positively addressing each question raised in Table 1

Figure 3- Decision tree of immunogenicity-related issues to address in a SmPC of a biological product whose immunogenicity is a potential issue 


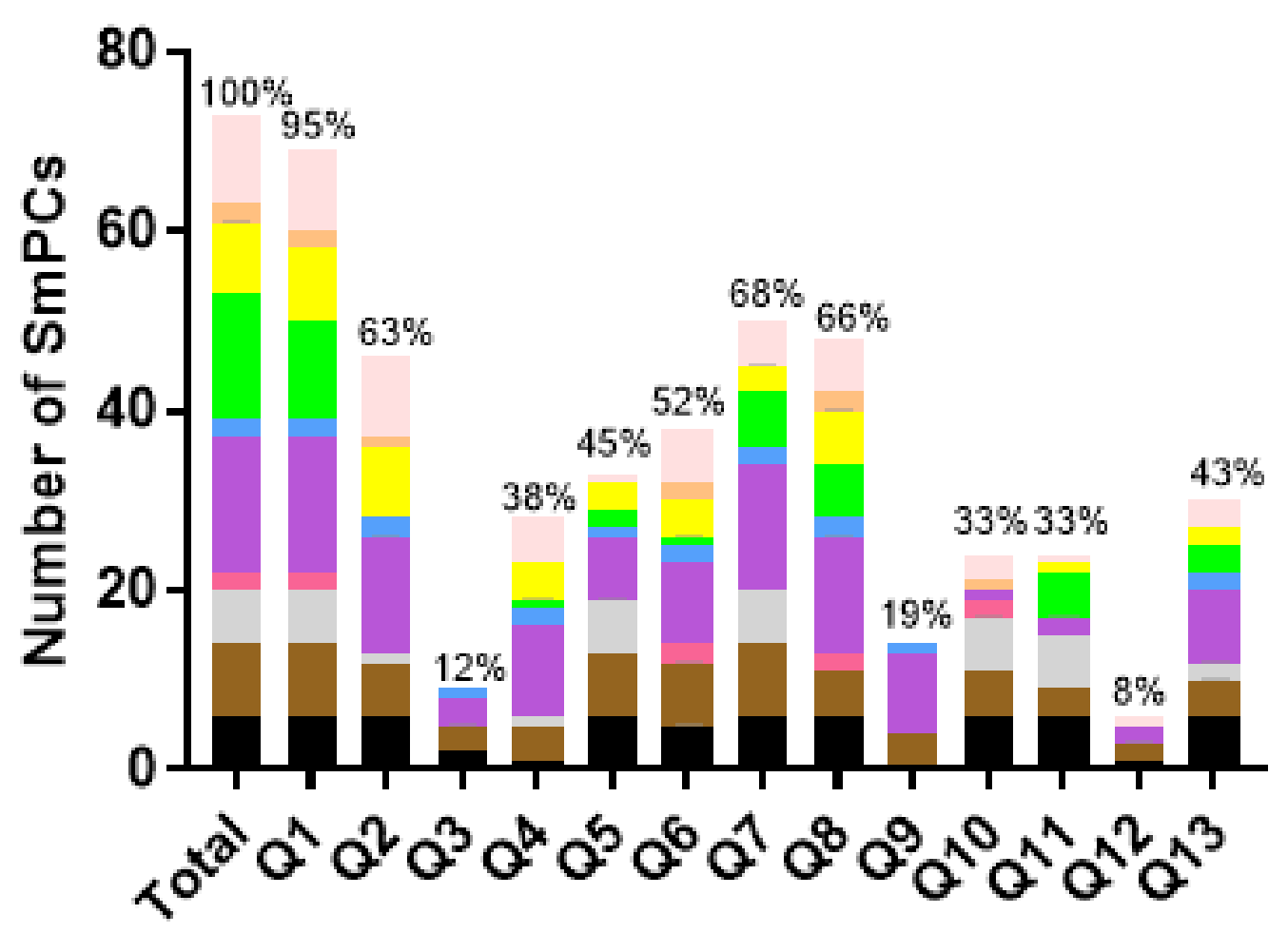

Positive answers to questions used to evaluate SmPCs
Other

Somatropins

Interferons

Insulins

Fusion Proteins

- Monoclonal Antibodies

- Filgrastims

Epoetins

Enzymes

- Antihaemophilic Factors 


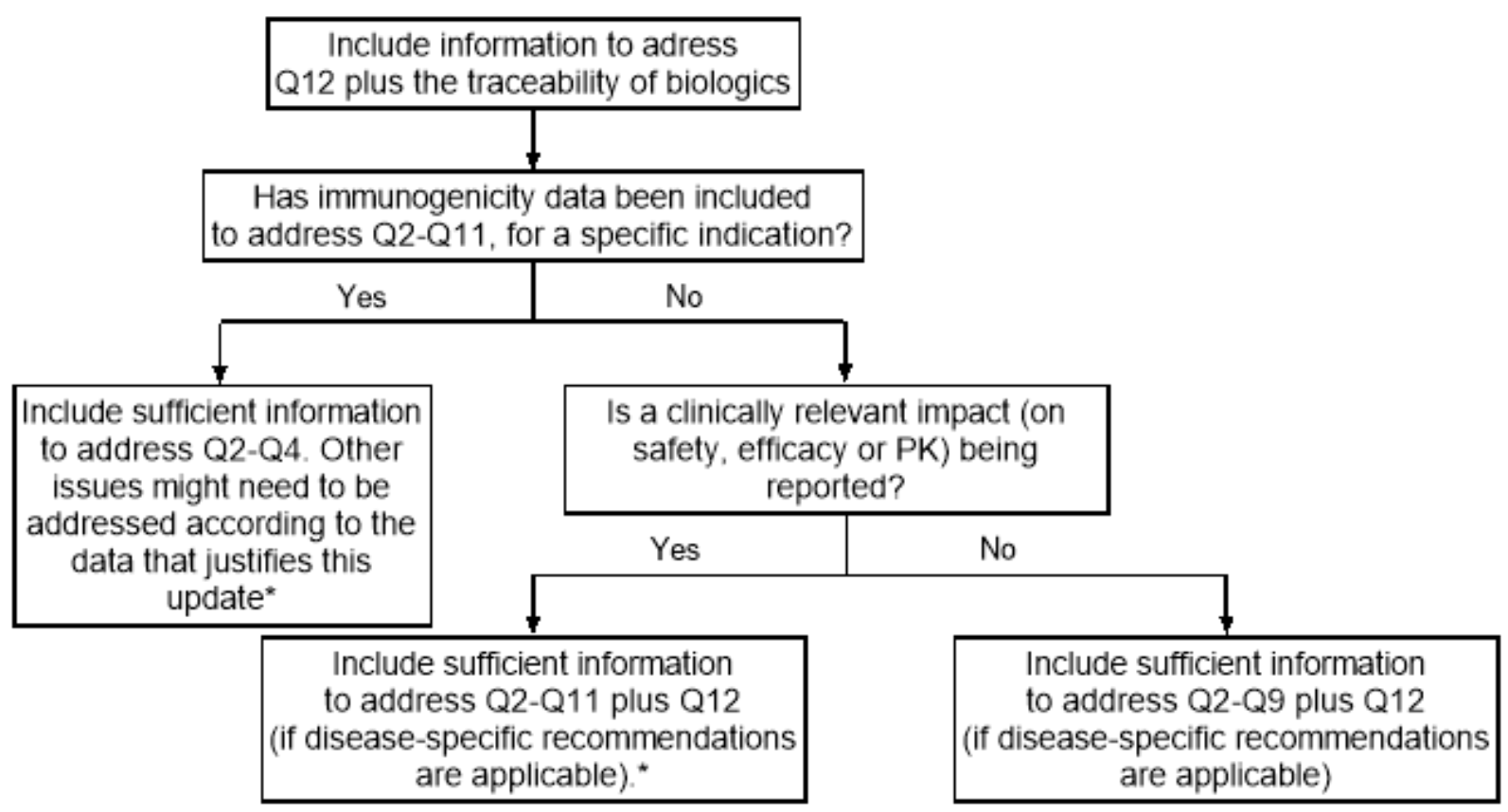

